\title{
Correlation of Circulating Matrix Metalloproteinase-3 and Osteopontin Levels with Postmenopausal Osteoporosis
}

\section{Yi DAI*}

Department of Orthopaedics and Trauma, Wuhan Hospital of Traditional Chinese Medicine, Wuhan, 430014, China

\begin{abstract}
Objective: To study Matrix Metalloproteinase-3 (MMP-3) and Osteopontin (OPN) levels and correlations of MMP3 and OPN with Bone Metabolic Markers and Bone Mineral Density (BMD) in postmenopausal Chinese women.

Methods: MMP-3, OPN Osteoprotegrin (OPG), Osteoprotegrin Ligand (OPGL) of 120 postmenopausal Chinese female volunteers was measured using ELISA. BMD were measured using dual energy X-ray absorptiometry. According to the criteria of $\mathrm{WHO}$, women were divided into 3 groups, such as normal, oteopenia and osteoporosis group.

Results: OPN concentrations were significantly higher in osteoporosis $(56 \pm 20) \mathrm{ng} / \mathrm{ml}$ than normal $(26 \pm 11)$ $\mathrm{ng} / \mathrm{ml}(\mathrm{P}<0.05)$. But MMP-3 concentrations were little higher in osteoporosis $(153 \pm 121) \mathrm{ng} / \mathrm{ml}$ than normal $(125 \pm$ 101) $\mathrm{ng} / \mathrm{ml}$.

In osteoporosis, notable negative correlations between OPN, ratio of OPN/MMP-3 and BMD, SOPGL were found $(P<0.05)$ as well as positive relations between OPN, ratio of OPN/MMP-3 and SOPG $(P<0.05)$, and positive relation of MMP-3 and BMD of Ward's triangle was existed $(P<0.05)$, in osteopenia negative relations of MMP-3 and BMD as well as ratio of OPN/MMP-3 were detected $(P<0.05)$.Conclusion: There are significant correlations between serum OPN, ratios of OPN and MMP-3 and bone biomarkers of SOPG, sOPGL, OPN and OPN/MMP-3 may increase with high bone-metabolism. The increases of OPN and ratio of OPN/MMP-3 appear possibly as a concomitant event in high bone turnover state, such as postmenopausal osteoporosis.
\end{abstract}

Keywords: Matrix metalloproteinase-3; Osteopontin; Bone mineral density Postmenopausal-osteoporosis

Osteoporosis is a metabolic condition characterized by decreased bone mass and strength due to increased bone turnover, which compromises bone architecture and results in increased fracture risk. Postmenopausal osteoporosis is the most common type of osteoporosis associated with estrogen deficiency [1]. However, the mechanisms by which estrogen deficiency causes bone loss are complex and are not fully understood.

Matrix metalloproteinase (Matrix Metalloproteinase, MMP) forms a family of zinc-dependent proteinases essential for several physiological and pathological events, such as embryonic development, angiogenesis, wound repair, periodontal disease, rheumatoid arthritis, cancer invasion, and metastasis. Osteoblast-derived MMPs have been shown to play a role in bone metabolism by degradation of the bone matrix. Bone resorption is dependent on the activity MMP-3, which degrades denatured type II collagen and other components of the bone organic matrix. Evidence has accumulated for an active participation by osteoblast-derived MMPs in the initiation of bone resorption and coupled bone formation by degrading the unmineralized osteoid layer of the bone surface to allow osteoclasts to attach to the mineralized matrix. In vitro [2,3], normal osteoblasts secrete abundant MMP-3 which was triggered by osteopontin (OPN), then OPNMMP-3 complex were existed to induce bone matrix into ossification before bone mineralization.

\section{Methods}

\section{Subjects}

The study population comprised 120 postmenopausal women aged 48-65 years, selected randomly from Wuhan, China and its surrounding area. All subjects were screened by means of a detailed questionnaire, medical history taking, and physical examination. Menopause was defined as 1 year with no menstrual flow.
Subjects were excluded from the study if they had oligomenorrhea or a menorrhea before the age of 40 years or if they had such conditions affecting bone metabolism as diseases of the kidney, liver, parathyroid, or thyroid, diabetes mellitus, hyperprolactinemia, oophorectomy, rheumatoid arthritis, ankylosing spondylitis, malabsorption syndromes, malignant tumors, hematologic diseases, previous pathological fractures, hypertension, coronary artery disease, angiopathy, myocardial infarction, cerebral infarction, and infectious disease. If the subjects had received treatment with glucocorticoids, estrogens, thyroid hormone, parathyroid hormone, fluoride, bisphosphonate, calcitonin, thiazide diuretics, barbiturates, anti-seizure medication, vitamin D and calcium-containing drugs, they were also excluded. Body height and weight were measured using a stadiometer and a standardized balancebeam scale, respectively. This study was approved by the Research Ethics Committee of Wuhan Union Hospital, and informed consent was obtained from each woman before the study.

Among the postmenopausal women, 40 were considered osteoporosis according to the WHO criterion: Bone Mineral Density (BMD) more than $2.5 \mathrm{SD}$ below the normal adult mean based on the established reference databases. 40 women whose BMD was less than $1.0 \mathrm{SD}$ below the normal were included into the normal group.40 volunteers were osteopnia whose BMD were between $2.5 \mathrm{SD}$ and $1.0 \mathrm{SD}$.

*Corresponding author: Yi Dai, Department of Orthopaedics and Trauma, Wuhan Hospital of Traditional Chinese Medicine, Wuhan, 430014, China, E-mail: daiyiwh@163.com

Received May 12, 2013; Accepted June 26, 2013; Published June 28, 2013

Citation: Yi DAI (2013) Correlation of Circulating Matrix Metalloproteinase-3 and Osteopontin Levels with Postmenopausal Osteoporosis. J Trauma Treat 2: 171 doi:10.4172/2167-1222.1000171

Copyright: (c) 2013 Yi DAI. This is an open-access article distributed under the terms of the Creative Commons Attribution License, which permits unrestricted use, distribution, and reproduction in any medium, provided the original author and source are credited. 


\section{Metabolic assays}

Blood samples were collected between 7:00 a.m. and 8:30 a.m. after fasting overnight and allowed to clot. Thereafter the samples were centrifuged and divided into aliquots and stored at $-70^{\circ} \mathrm{C}$ until being assayed. The serum concentration of OPN, MMP-3, OPG and OPGL was measured with ELISA kits (Boster Biosciences Co., Wuhan, China). The intra- and inter-assay CV were less than $5.6 \%$ and $6.2 \%$ for OPN, $6.5 \%$ and $6.0 \%$ for MMP-3, 5.4\% and $6.9 \%$ for OPG, $6.5 \%$ and $6.7 \%$ for OPGL, respectively. Serum OPG levels were measured using ELISA kits (Boster Biosciences Co., Wuhan, China). Then the ratio of OPN to MMP-3(list by OPN/MMP-3) and OPG to OPGL (list by OPG/OPGL) were calculated.

\section{Measurements of BMD}

BMD at the lumbar spine (L1-L4), the left hip including the femoral neck, trochanter and Ward's triangle was measured by Dual Energy X-Ray Absorptiometry (DEXA) using a Lunar-prodity device (GE, Mass, USA).

All BMD results were expressed as $\mathrm{g} / \mathrm{cm}^{2}$. The precision of the DXA was evaluated over three repeated measurements of different BMD values in subjects, giving a mean coefficient of variation (CV) (range: $0.33 \%-0.40 \%$ ) [4]. Phantom scan over the control spine was performed everyday with a long-term (more than 5 years) CV of $0.33 \%-0.40 \%$ and a root-mean-square coefficient of variation of $0.35 \%$ (degrees of freedom $(\mathrm{df})=1,482)$.

\section{Statistical analysis}

Statistical analysis was made with the SPSS 13.0 software. The results were expressed as mean \pm Standard Deviation (SD). The correlations between OPN, MMP-3 and OPG, OPGL, BMD were assessed by Pearson's product-moment correlation coefficient and partial correlation analysis after adjustment for age and body mass index (BMI). Comparisons were made by one-way ANOVA. P value less than 0.05 was considered statistically significant.

\section{Results}

\section{Characteristics}

Among the 120 postmenopausal women, 40 age-matched normal controls, 40 age-matched osteopenia and 40 age-matched osteoporotic women were compared for the concentrations of Characteristics (Table 1) and serum OPN and MMP-3 (Table 2).

Among groups, there was no significant difference in age, BMI, and years after menopause $(\mathrm{P}>0.05)$. Table 2 shows that the concentrations of serum MMP-3, OPN and the ratio of OPN to MMP-3 were significantly higher in women with osteoporosis than in age-matched normal controls $(\mathrm{P}<0.01)$. The concentrations of serum OPG were significantly higher in osteoporosis than normal controls $(\mathrm{P}<0.05)$ The BMD at various skeletal sites and serum calcium concentration in women with osteoporosis were significantly lower than those in normal controls $(\mathrm{P}<0.05)$.

\section{Correlation of OPN, MMP-3 and OPN/MMP-3 with bone metabolism marker and $\mathrm{BMD}$}

Significant negative correlations of OPN and MMP-3 with BMD and OPGL were observed in all skeletal regions, and positive correlations of OPN and MMP-3 with OPG and OPG/OPGL were seen. After adjustment for age and body mass index (BMI), the above correlations were decreased but remained significant (Table 3 ).

In osteopenia, only significant correlations of MMP-3 with BMD of lumber spine and ward angle were detected. In osteoporosis group, significant correlations of MMP-3 and ratio of OPN to MMP-3 with OPG, OPGL and BMD in all skeletal regions were detected (Table 4).

\section{Discussion}

OPG-OPGL system was associated with activating and differentiating of osteoclasts in osteoporosis. Over-expression of OPG in transgenic mice might lead to severe osteopetrosis associated with a decrease in osteoclasts in metaphyseal trabecular bone [5]. In contrast, OPG-deficient mice exhibited an overall decrease in total bone density and a high incidence of fractures, reminiscent of postmenopausal osteoporosis in human populations [6]. As the same time, the ratio of OPG to OPGL was more relative with estrogen than OPG and OPGL. In our study, levels of OPG, OPGL and the ratio of OPG to OPGL in osteoporosis were more than the normal, so that postmenopausal women of osteoporosis were in high turn-over bone metabolism statue. MMP could degrade extra-cellar matrix of bone, especially in postmenopausal osteoporosis. In low estrogen level, MMP would activate osteoclasts maturing, immigrating and sticking according to degrading the matrix [7].

MMP-3, also known as stromlysin-1, was secreted by osteoblasts, when estrogen was deficient, osteoblast was stimulated by osteoclasts, and therefore MMP-3 was much expressed in matrix around osteocyte and its lacuna in order to participate in collagen II and cartilage metabolism. In past, MMP-3 was considered to degrading noncollagen, such as proteoglycan not to act on collagen I metabolism $[2,8]$, but trigger bone resorption and formation. In recent [9], matrix metalloproteinases family was high linked to small integrin-binding

\begin{tabular}{|c|c|c|c|c|c|c|}
\hline & Case & Age (yr) & Hight (cm) & Weight (kg) & BMI(kg/m $\mathbf{~}^{\mathbf{2}}$ & Years after menopause (yr) \\
\hline Normal & 40 & $52.36 \pm 2.56$ & $156 \pm 6$ & $53 \pm 3$ & $23.16 \pm 2.58$ & $3.87 \pm 1.36$ \\
\hline Osteopenia & 40 & $52.98 \pm 3.64$ & $155 \pm 4$ & $56 \pm 4$ & $22.26 \pm 3.04$ \\
\hline Osteoporosis & 40 & $53.83 \pm 2.17$ & $154 \pm 6$ & $55 \pm 4$ & $22.88 \pm 2.76$ \\
\hline
\end{tabular}

Table 1: List of Characteristic $(\bar{x} \pm \mathrm{S})$

\begin{tabular}{|c|c|c|c|c|c|c|}
\hline & OPG(U/L) & OPGL $(\mu \mathrm{g} / \mathrm{L})$ & OPG / OPGL & MMP3( $\mu \mathrm{g} / \mathrm{L})$ & OPN(ng/mL) & OPN/MMP-3 \\
\hline Normal & $62.21 \pm 2.25$ & $6.31 \pm 0.51$ & $9.38 \pm 1.13$ & $125 \pm 101$ & $26 \pm 11$ & $0.21 \pm 0.11$ \\
\hline Osteopenia & $113.31 \pm 11.42^{*}$ & $4.82 \pm 0.41^{*}$ & $23.42 \pm 3.26$ & $134 \pm 122$ & $36 \pm 15$ & $0.26 \pm 0.12$ \\
\hline Osteoporosis & $128.61 \pm 5.25^{*}$ & $3.82 \pm 0.21^{*}$ & $32.59 \pm 3.26^{* \Delta}$ & $153 \pm 121^{* \Delta}$ & $56 \pm 20^{\star \Delta}$ & $0.36 \pm 0.16^{* \Delta}$ \\
\hline
\end{tabular}

${ }^{*}$ Compared to Normal $P<0.05,{ }^{\wedge}$ Compared to osteopenia $P<0.05$ 


\begin{tabular}{|c|c|c|c|c|c|c|c|c|}
\hline & & \multicolumn{4}{|c|}{ BMD } & \multicolumn{3}{|c|}{ Bone Biomarker } \\
\hline & & Lumber spine & Femoral neck & Trochanter & Ward's triangle & OPG & OPGL & OPG/OPGL \\
\hline \multirow{3}{*}{$\begin{array}{c}\text { Un- } \\
\text { adjusted }\end{array}$} & MMP3 & $-0.197^{*}$ & -0.134 & -0.111 & $-0.221^{*}$ & $0.201^{*}$ & $-0.194^{*}$ & $0.205^{*}$ \\
\hline & OPN & $-0.208^{*}$ & -0.137 & -0.125 & $-0.202^{*}$ & $0.198^{*}$ & $-0.191^{*}$ & $0.198^{*}$ \\
\hline & ratio of OPN/MMP-3 & $-0.255^{\star}$ & $-0.209^{*}$ & -0.113 & $-0.236^{*}$ & $0.219^{*}$ & $-0.208^{*}$ & $0.213^{*}$ \\
\hline \multirow{3}{*}{$\begin{array}{l}\text { Adjusted by Age } \\
\text { and BMI } \\
{ }^{*} P<0.05\end{array}$} & MMP3 & -0.175 & -0.117 & -0.103 & $-0.213^{*}$ & $0.196^{*}$ & $-0.162^{*}$ & $0.201^{*}$ \\
\hline & TIMP1 & -0.186 & -0.121 & -0.116 & $-0.208^{*}$ & 0.175 & -0.156 & 0.172 \\
\hline & ratio of OPN/MMP-3 & $-0.201^{*}$ & $-0.196^{*}$ & -0.105 & $-0.209^{*}$ & $0.203^{*}$ & $-0.197^{*}$ & $0.193^{*}$ \\
\hline
\end{tabular}

Table 3: Relation of Serum Level of MMP3, OPN and Bone Biomarker in Postmenopausal.

\begin{tabular}{|c|c|c|c|c|c|c|c|c|}
\hline & & \multicolumn{4}{|c|}{ BMD } & \multicolumn{3}{|c|}{ Bone Biomarker } \\
\hline & & Lumber spine & Femoral neck & Trocha-nter & Ward's triangle & OPG & OPGL & OPG/OPGL \\
\hline \multirow{3}{*}{ Osteopenia } & MMP3 & $-0.188^{*}$ & -0.129 & -0.084 & $-0.196^{*}$ & 0.169 & -0.152 & 0.124 \\
\hline & OPN & 0.159 & 0.133 & 0.057 & 0.135 & -0.155 & 0.115 & -0.129 \\
\hline & ratio of OPN/MMP-3 & -0.126 & -0.124 & -0.079 & $-0.187^{*}$ & 0.181 & -0.138 & 0.165 \\
\hline \multirow{3}{*}{$\begin{array}{l}\text { Osteoporosis } \\
{ }^{*} P<0.05\end{array}$} & MMP3 & $-0.296^{*}$ & $-0.198^{*}$ & -0.121 & $-0.301^{*}$ & $0.228^{*}$ & $-0.233^{*}$ & $0.285^{\star}$ \\
\hline & OPN & 0.178 & 0.165 & 0.108 & $0.198^{*}$ & -0.165 & 0.137 & -0.146 \\
\hline & ratio of OPN/MMP-3 & $-0.301^{*}$ & $-0.208^{*}$ & -0.135 & $-0.309^{*}$ & $0.223^{*}$ & $-0.252^{*}$ & $0.354^{*}$ \\
\hline
\end{tabular}

Table 4: Relation of Serum Level of MMP3, OPN and Bone Biomarker in osteopenia and osteoporosis

ligand, N-linked glycoprotein family. The latter who combined with and activated the former take great part in the mineralization of bone.

Osteopontin (OPN) is a $34-\mathrm{kDa}$ acidic, secreted phosphoprotein that was originally identified as a major component of the noncollagenous bone matrix expressed in both osteoblasts and osteoclasts. It has been found to be associated with bone strength and bone remodeling. Previous reports have indicated that OPN knockout mice are resistant to ovariectomy-induced bone resorption compared with wild-type mice. It have been proved that OPN were much more relatives to osteoporosis [3]. In our study [9], OPN in osteoporosis was much higher than in normal, so that postmenopausal women of osteoporosis were in high turn-over bone metabolism statue.

In pre-matured bone tissue, non-collagen was modified by OPNMMP-3-complexity before bone matrix was mineralized in order to maintain local micro circumstance by protein-lyses of complexity. It was in osteoporotic metabolism triggered by osteoclasts that osteoblasts was crawling and surviving in mineralizing and high bone turn-over situation $[10,11]$. In our case, levels of MMP-3 and OPN in osteoporosis were high than the normal and both were relatives with bone mineral density and OPG/OPGL. It was shown that MMP-3 was probably the triggered factor of postmenopausal osteoporosis and OPN-MMP-3 complexity was probably the bridge to explore the onset of PMOP.

\section{Conclusion}

Serum MMP-3 and OPN/MMP-3 would be the determinant factors of postmenopausal osteoporosis. They are correlated with markers of bone metabolism and their concentrations are increased in boneturnover. They might be changed as a concomitant bone metabolic event in bone metabolism such as postmenopausal osteoporosis.

\section{Acknowledgements}

This work was supported by grants from Foundation of Wuhan Youth Science Sunlight Project (200950431215), Hubei Health Youth Project (QJX2010-48) and Wuhan Health Study Project (WZ10C02).

\section{References}

1. Manolagas SC (2000) Birth and death of bone cells: basic regulatory mechanisms and implications for the pathogenesis and treatment of osteoporosis. Endocr Rev 21: 115-137.

2. Breckon JJ, Papaioannou S, Kon LW, Tumber A, Hembry RM, et al. (1999) Stromelysin (MMP-3) synthesis is up-regulated in estrogen-deficient mouse osteoblasts in vivo and in vitro. J Bone Miner Res 14: 1880-1890.

3. Huang W, Carlsen B, Rudkin G, Berry M, Ishida K, et al. (2004) Osteopontin is a negative regulator of proliferation and differentiation in MC3T3-E1 preosteoblastic cells. Bone 34: 799-808.

4. Wu XP, Liao EY, Zhang H, Shan PF, Cao XZ, et al. (2004) Establishment of BMD reference plots and determination of peak BMD at multiple skeletal regions in mainland Chinese women and the diagnosis of osteoporosis. Osteoporos In 15: $71-79$

5. Minenna G, D'Amore S, Maggiolini P, Scagliusi P, D'Amore M (2005) [RANKL/ RANK, OPG and OPT in a group of patients affected by chronic arthritis. Preliminary report]. Recenti Prog Med 96: 431-432.

6. Dai Y, Shen L (2007) Relationships between serum osteoprotegerin, matrix metalloproteinase-2 levels and bone metabolism in postmenopausal women. Chin Med J (Engl) 120: 2017-2021.

7. Dai Y, Shen L (2007) Association of circulating matrix metalloproteinase-2 and tissue inhibitor of matrix metalloproteinase-2 levels with bone metabolic markers and bone mineral density in Chinese postmenopausal women. Zhonghua Neifenmi Daixie Zazhi. s23: 06-210.

8. Manicourt DH, Fujimoto N, Obata K, Thonar EJ (1995) Levels of circulating collagenase, stromelysin-1, and tissue inhibitor of matrix metalloproteinases 1 in patients with rheumatoid arthritis. Relationship to serum levels of antigenic keratan sulfate and systemic parameters of inflammation. Arthritis Rheum 38: 1031-1039.

9. Shapses SA, Cifuentes M, Spevak L, Chowdhury H, Brittingham J, et al. (2003) Osteopontin facilitates bone resorption, decreasing bone mineral crystallinity and content during calcium deficiency. Calcif Tissue Int 73: 86-92.

10. Ogbureke KU, Fisher LW (2004) Expression of SIBLINGs and their partner MMPs in salivary glands. J Dent Res 83: 664-670.

11. Fedarko NS, Jain A, Karadag A, Fisher LW (2004) Three small integrin binding ligand N-linked glycoproteins (SIBLINGs) bind and activate specific matrix metalloproteinases. FASEB J 18: 734-736. 